\title{
ANÁLISIS DE ACTIVOS FINANCIEROS UTILIZANDO HERRAMIENTAS ESTADÍSTICAS: DESVIACIÓN TÍPICA Y COEFICIENTE DE CORRELACIÓN
}

\author{
ANALYSIS OF ASSETS FINANCIAL USING STATISTICAL TOOLS: \\ STANDARD DEVIATION AND CORRELATION COEFFICIENT
}

\author{
Nicko A. Gomero Gonzales * \\ Docente Asociado de la Facultad de Ciencias Contables, UNMSM
}

[Recepción: Enero de 2010 / Conformidad: Mayo de 2010]

\section{RESUMEN}

Tomar decisiones financieras que conlleven a minimizar riesgos, implica manejar una serie de instrumentos económicos, matemáticos o estadísticos. Bajo esta óptica, se puede afirmar que el inversionista, llamado coberturador o especulador, estará en mejor posición con su cartera, si para su selección ha utilizado los más variados instrumentos que brindan las diferentes disciplinas científicas. Ya sea el análisis técnico o fundamental amerita el uso de estas herramientas, con lo cual las decisiones serán mucho más acertadas, esta exigencia debe darse con mucho mayor rigurosidad dada las características de la interdependencia de los mercados. Por ello, a continuación, se presenta al lector una nota metodológica para el uso de dos herramientas estadísticas básicas para optar por la mejor posición financiera: La desviación típica y el coeficiente de correlación.

Palabras claves: Cartera de activos financieros, portafolio, riesgo, desviación típica, correlación, rentabilidad esperada, acciones, bonos.

\begin{abstract}
To make financial decisions that bring to minimizing risks, involves managing a number of economic instruments, mathematical or statistical. From this viewpoint, we can say that the investor call speculator will be better positon with his portfolio, if your selection has been used for the most varied instruments offered by the different scientific disciplines. Either technical or fundamental analysis requires the use of these tools, with these the decisions acceptable, this requirement should be given far more rigorously the characteristics of market interdependence. So then presents the reader with a methodological note for the use of two basic statistical tools to choose the best financial position: The standard deviation and coefficient of correlation.
\end{abstract}

Key words: Financial asset portfolio, portfolio, risk, deviation, correlation, expected returns, stocks, bonds.

\footnotetext{
* Doctor en Ciencias Económicas. Docente de la Unidad de Posgrado y Docente Asociado de la Facultad de Ciencias Contables, UNMSM. E-mail: nickgo_6@msn.com, gomero_econ@yahoo.es
} 


\section{INTRODUCCIÓN}

Bajo el proceso de la globalización, y con ello la mayor interdependencia económica y financiera, los mercados bursátiles han tendido a volatilizarse en mayor magnitud, siendo una evidencia clara de este fenómeno la última crisis internacional, cuyos efectos aún siguen latentes en muchos países y con síntomas de permanecer por un tiempo prolongado que inclusive el FMI no ha acertado con precisión en su pronóstico. La crisis griega es una clara señal que los mercados siguen volatilizados, con características de globalizarse pero con mayor profundidad, efectos que se pueden tipificar como estructurales y que son propios del proceso de integración de los mercados.

La elevada sensibilización de los mercados bursátiles, exige al inversionista estar mejor coberturado, si bien, para este fin podría optar por la diversificación de su cartera, tal como señala la teoría de portafolio de Markowitz o, en todo caso, podría utilizar la teoría del CAMP para elegir una cartera con activos financieros de altas y bajas BETAS o también podría optar por estructurar una cartera cuya volatilidad medida a través del Beta, tienda a ser igual al del mercado.

En fin, las disciplinas científicas brindan al inversionista, una serie de herramientas y teorías que forzosamente tienen que ser utilizados cuando se desea tomar decisiones con elevadas probabilidades de acierto. Como los mercados cada día se volatilizan más, estos nuevos escenarios exigen la utilización de nuevas herramientas para optar por la mejor posición en el campo financiero, especialmente cuando se trata de activos de alta sensibilidad. Para este fin, se presenta al lector dos herramientas estadísticas básicas, como son el coeficiente de correlación y la desviación típica, que siguiendo un proceso metodológico serán utilizados para medir riesgos y rendimientos de dos activos financieros: bonos $y$ acciones.

\section{Desviación típica y valuación de bonos}

Si un inversionista posee un perfil de aversión al riesgo puede comprar bonos de la más alta calificación, que le conllevaría lograr tasas libres de riesgo. Estos tipos de bonos mayormente son los gubernamentales o los emitidos por las autoridades monetarias, bajo el respaldo de un riesgo país que proyecte confiabilidad total en sus obligaciones de largo plazo. Cuando se trata de bonos que comúnmente se le denomina activo financiero de renta fija, el BETA de los interés que ha de pagar durante el periodo de vigencias va a ser igual a cero, pero no es así para su precio de negociación en el mercado secundario, ya que su valor dependerá de las fuerzas del mercado.

Como los intereses de un bono están bajo contrato, su grado de volatilidad es igual a cero, pero esta condición no se cumple para el valor de su cotización, cuya desviación típica o sensibilidad va a depender de la tasa de interés del mercado. Aquí una demostración matemática:

Sea un bono gubernamental con el siguiente perfil:

- Precio de emisión: US\$10,000

- Tasa de emisión: $10 \%$

- Periodo de vigencia: 10 años

- Cantidad de bonos emitidos: 10,000

- Calificación del bono: A+ (nulo riesgo de incumplimiento de pago)

$\mathrm{El}$ inversionista del título recibirá por 10 años US\$ 1000 de intereses, pero al vencimiento recuperará lo invertido, que dependerá de la cantidad de títulos que haya comprado en el mercado primario de valores. 
Aquí se demuestra que la sensibilidad de los intereses es igual a cero, pero no así para el precio de negociación en el mercado secundario, cuyo valor dependerá de los tipos de interés de mercado, que a continuación se detallan:

\begin{tabular}{|c|c|}
\hline $\begin{array}{c}\text { TASA DE EMISIÓN } \\
\text { (Tasa de interés que } \\
\text { paga el bono) }\end{array}$ & $\begin{array}{c}\text { TASA DE INTERÉS } \\
\text { (Mercado financiero) }\end{array}$ \\
\hline $10 \%$ & $7 \%$ \\
\hline $10 \%$ & $10 \%$ \\
\hline $10 \%$ & $15 \%$ \\
\hline
\end{tabular}

El principio financiero básico, teniendo en cuenta las tasas de interés señaladas, es la siguiente:

- Si la tasa de mercado es mayor a la tasa de emisión, el precio de negociación del bono en el mercado de valores será menor al precio de emisión.

- Si la tasa de mercado es menor a la tasa de emisión, el precio de negociación del bono en el mercado de valores será mayor al precio de emisión.

- Si la tasa de mercado es igual a la asa de emisión, el precio de negociación del bono en el mercado de valores será igual al precio de emisión.

En la tabla que se presenta a continuación se señalan los intereses que pagará el emisor del bono en diferentes periodos de tiempo y el momento en la cual se decide negociar este título en el mercado secundario. Por ejemplo, si se decide vender el bono después de un año, el precio en el mercado de valores será de $\$ 11,954.57$ o $\$ 7,614.21$, este valor dependerá de la tasa de interés de mercado; en el primer caso, la tasa vigente será del $7 \%$, y en el segundo 15\%. Estos precios fueron determinados utilizando el aplicativo EXCEL.

\begin{tabular}{|c|c|c|c|c|c|c|c|c|c|c|}
\hline AÑ̃S & $\mathbf{I}+\mathbf{A}$ & $\mathbf{I}+\mathbf{A}$ & $\mathbf{I}+\mathbf{A}$ & $\mathbf{I}+\mathbf{A}$ & $\mathbf{I}+\mathbf{A}$ & $\mathbf{I}+\mathbf{A}$ & $\mathbf{I}+\mathbf{A}$ & $\mathbf{I}+\mathbf{A}$ & $\mathbf{I}+\mathbf{A}$ & $\mathbf{I}+\mathbf{A}$ \\
\hline 1 & 1000 & & & & & & & & & \\
\hline 2 & 1000 & 1000 & & & & & & & & \\
\hline 3 & 1000 & 1000 & 1000 & & & & & & & \\
\hline 4 & 1000 & 1000 & 1000 & 1000 & & & & & & \\
\hline 5 & 1000 & 1000 & 1000 & 1000 & 1000 & & & & & \\
\hline 6 & 1000 & 1000 & 1000 & 1000 & 1000 & 1000 & & & & \\
\hline 7 & 1000 & 1000 & 1000 & 1000 & 1000 & 1000 & 1000 & & & \\
\hline 8 & 1000 & 1000 & 1000 & 1000 & 1000 & 1000 & 1000 & 1000 & & \\
\hline 9 & 1000 & 1000 & 1000 & 1000 & 1000 & 1000 & 1000 & 1000 & 1000 & \\
\hline 10 & 11000 & 11000 & 11000 & 11000 & 11000 & 11000 & 11000 & 11000 & 11000 & 11000 \\
\hline $7 \%$ & $\$ 12,107.07$ & $\$ 11,954.57$ & $\$ 11,791.39$ & $\$ 11,616.79$ & $\$ 11,429.96$ & $\$ 11,230.06$ & $\$ 11,016.16$ & $\$ 10,787.29$ & $\$ 10,542.41$ & $\$ 10,280.37$ \\
\hline $10 \%$ & $\$ 10,000.00$ & $\$ 10,000.00$ & $\$ 10,000.00$ & $\$ 10,000.00$ & $\$ 10,000.00$ & $\$ 10,000.00$ & $\$ 10,000.00$ & $\$ 10,000.00$ & $\$ 10,000.00$ & $\$ 10,000.00$ \\
\hline $15 \%$ & $\$ 7,490.62$ & $\$ 7,614.21$ & $\$ 7,756.34$ & $\$ 7,919.79$ & $\$ 8,107.76$ & $\$ 8,323.92$ & $\$ 8,572.51$ & $\$ 8,858.39$ & $\$ 9,187.15$ & $\$ 9,565.22$ \\
\hline I ( intereses) & 1000 & 1000 & 1000 & 1000 & 1000 & 1000 & 1000 & 1000 & 1000 & 1000 \\
\hline
\end{tabular}

Grado de volatilidad del precio de los bonos y de los intereses

\begin{tabular}{|l|c|c|c|c|}
\hline Tasa de interés e intereses que paga el bono & $7 \%$ & $10 \%$ & $15 \%$ & Intereses \\
\hline Desviación típica (riesgo) & 614.14 & 0 & 696.12 & 0 \\
\hline
\end{tabular}


Precio e interés de un bono en dólares

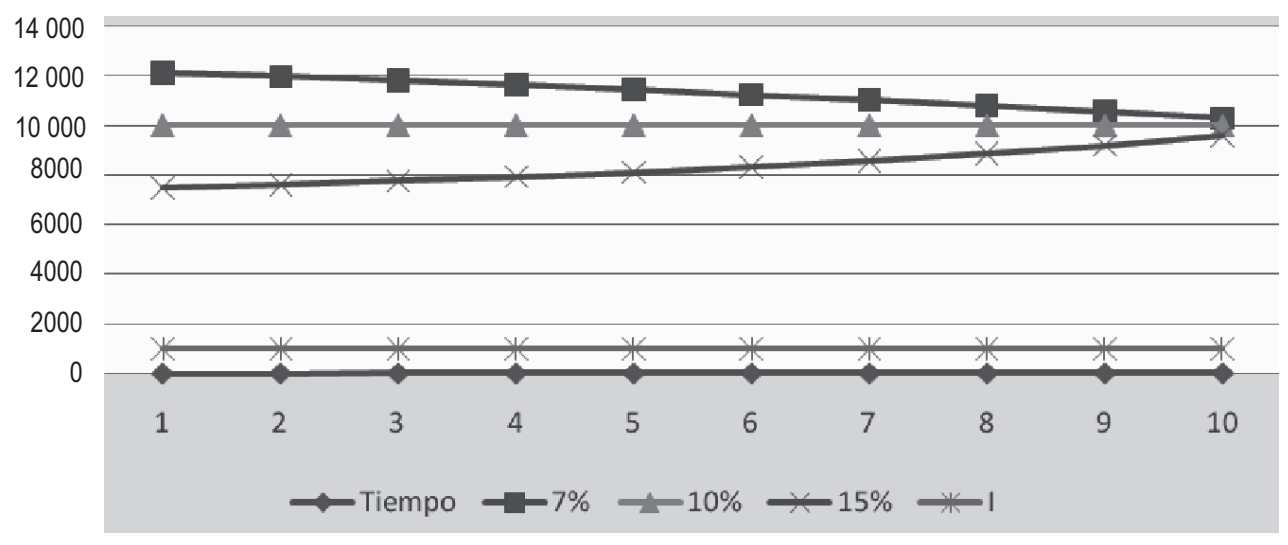

Los resultados indican que, cuando el precio del bono es igual a la par, su grado de volatilidad es cero, lo cual es lógico, ya que el mercado le asignará un valor igual al precio emitido, pero si las tasas de mercado difieren a la tasa de emisión, su grado de volatilidad medido a través de la desviación típica será mayor a cero. Por otro lado, como los intereses no tienen ninguna variabilidad en el tiempo la desviación típica será igual al cero.

Asimismo, por los resultados obtenidos se aprecia que, a medida que se va acercando la fecha de liquidación del bono, cualesquiera sea la tasa de mercado y el tiempo que queda por cobrar los intereses, el precio que se ha de negociar en el mercado secundario tiende a igualarse al precio de emisión. Es decir, en el último día de vigencia del bono, si se quiere vender o comprar en el mercado de valores, el precio asignado a este activo financiero será de US\$10,000.

En el caso presentando por una cuestión metodológica se está señalando que la tasa de interés cambia en forma anual, pero no necesariamente es así, debido a que el mercado monetario puede cambiar de un día a otro, o en todo caso en horas. Por ejemplo, el Banco Central puede cambiar o anunciar un cambio de la tasa de política monetaria o en todo caso puede tomar la decisión de aplicar una política monetaria expansiva o restrictiva, de ser así, los tipos de interés cambiarán automáticamente, induciendo con ello a que cambien de posición los especuladores con su portafolio en el mercado de capitales. En este sentido, se puede afirmar que la volatilidad del precio de los bonos va a depender de la magnitud y frecuencia de cambio de los tipos de interés del mercado monetario, con ello la desviación típica tendería a ser mayor.

\section{Desviación típica y correlación en la valuación de acciones}

Cuando se trata de acciones, también es de utilidad la desviación típica para tomar decisiones financieras, la regla es la siguiente:

- Cuanto más volátil es una acción en el mercado secundario, su desviación típica será mayor, por lo que el inversionista esperará un mayor rendimiento

- Cuanto menos volátil es la acción, su desviación típica será menor, por lo que en esta situación, el especulador esperará un menor retorno por su inversión.

La utilización de esta herramienta estadística se podría utilizar cuando se desea obtener la rentabilidad de una acción bajo diferentes condiciones económicas, tal como se 
plantea a continuación: el activo financiero de renta variable podría tener tres posibles rentabilidades, dependiendo este resultado del escenario económico vigente, y de la probabilidad de ocurrencia (Pi).

Para llegar a cuantificar el riesgo bajo las condiciones planteadas, primero hay que determinar el rendimiento esperado promedio $(\mathrm{Re})$, el cual es igual al rendimiento observado del título, multiplicado por su probabilidad de ocurrencia. Tomando en cuenta la característica del activo financiero la rentabilidad esperada será del $14.5 \%$.

\begin{tabular}{|c|c|c|c|}
\hline Escenario & $\begin{array}{c}\text { Rendimiento } \\
\text { del título } \\
\text { RI }\end{array}$ & $\begin{array}{c}\text { Probabilidad } \\
\text { de ocurrencia } \\
\text { Pi }\end{array}$ & $\begin{array}{c}\text { Rendimiento } \\
\text { esperado } \\
\text { Rei }\end{array}$ \\
\hline Alza & 0.3 & 0.5 & 0.15 \\
\hline Baja & 0.05 & 0.3 & 0.015 \\
\hline Crisis & -0.1 & 0.2 & -0.02 \\
\hline & \multicolumn{2}{|c|}{ Rendimiento esperado promedio del titulo } \\
0.145 $=14.5 \%$
\end{tabular}

El rendimiento esperado promedio es de utilidad para que el inversionista tome una adecuada posición financiera, el cual puede ser complementado con un análisis de riesgo, para ello se tiene que determinar la desviación típica del título, que estaría indicando, además, su grado de volatilidad, el cual es igual a $16.34 \%$, que bien podría ser considerado como una prima por riesgo.

Para obtener la rentabilidad del título considerando la prima por riesgo (desviación típica), es necesario utilizar la siguiente formula:

\section{$\operatorname{Re}=$ rentabilidad promedio + - desviación típica del título ( $68 \%$ de confianza)}

$\operatorname{Re}=$ rentabilidad promedio +-2 (desviación típica del título) (95\% de confianza)

A continuación, se presenta un cuadro donde se determina; la volatilidad del título.

\begin{tabular}{|c|c|c|c|c|c|c|}
\hline Escenario & $\begin{array}{c}\text { Rendimiento } \\
\text { del título } \\
\text { RI }\end{array}$ & $\begin{array}{c}\text { Probabilidad } \\
\text { de ocurrencia } \\
\mathbf{P i}\end{array}$ & $\begin{array}{c}\text { Rendimiento } \\
\text { Esperado } \\
\text { Rei }\end{array}$ & (Ri-Rei) & (Ri-Rei)2 & (Ri-Rei)2Pi \\
\hline Alza & 0.3 & 0.5 & 0.15 & 0.155 & 0.024025 & 0.012013 \\
\hline Baja & 0.05 & 0.3 & 0.015 & -0.095 & 0.009025 & 0.002708 \\
\hline Crisis & -0.1 & 0.2 & -0.02 & -0.245 & 0.060025 & 0.012005 \\
\hline & \multicolumn{7}{|r|}{ Desviación típica de la rentabilidad de la acción } & 0.026725 \\
\hline & \multicolumn{7}{|c|}{$\mathbf{0 . 1 4 5}$} \\
\hline
\end{tabular}

Tomando en cuenta la información obtenida, la rentabilidad máxima que esperaría el inversionista por este título sería del 30.84\% y la mínima llegaría al - 1.84\%. Al analizar la fórmula se puede llegar a establecer que:

"Cuanto mayor es la desviación típica del título, será más sensible o riesgosa, por lo que los rangos de rendimientos será mayor. El inversionista, bien podría obtener altas o bajas rentabilidades si posee títulos con esta característica”.
Otra herramienta estadística importante para; la toma decisiones en el campo bursátil es coeficiente de correlación. Este instrumento se operativiza cuando se quiere estructurar una cartera con dos tipos de títulos. Siendo la regla la siguiente:

Si dos títulos tienen una correlación positiva y cercana $a+1$, la cartera será de alto riesgo.

Si dos títulos tienen una correlación negativa y cercana $a-1$, será una cartera de bajo 
riesgo, con tendencia a una alta cobertura ante riesgos.

Por ejemplo, Supóngase que se cuenta con dos carteras de títulos con los siguientes coeficientes de correlación:

\begin{tabular}{|c|c|}
\hline Cartera & Correlación de dos títulos \\
\hline Cartera A & +0.70 \\
\hline Cartera B & -0.70 \\
\hline Cartera C & +1.0 \\
\hline Cartera D & -1.0 \\
\hline
\end{tabular}

El inversionista estará asumiendo un mayor riesgo si mantiene la cartera $\mathrm{C}$ y estará perfectamente coberturado cuando su cartera posee una correlación de $-1,0$ o sea la cartera D. Entre la cartera A y B , estará asumiendo mayor nivel de riesgo con la primera cartera. Tomando en cuenta esta herramienta estadística, un inversionista adverso al riesgo optará por carteras con correlaciones negativas pero cercanas a -1 , pero el amante al riesgo optará por estructurar portafolios con correlaciones positivas pero cercanas a + 1. La rentabilidad obtenida de los primeros, sería menor con relación a los que asumirían riesgos elevados o carteras con correlaciones positivas.

A continuación se presenta una tabla con rentabilidades observadas para 4 tipos de títulos, se desea saber que cartera sería la de mayor y bajo riesgo.

\begin{tabular}{|l|c|c|c|c|}
\hline \multicolumn{1}{|c|}{ Meses } & BNV & ATK & BNK & GRNC \\
\hline Enero & 0.15 & 0.12 & 0.1 & 0.05 \\
\hline Febrero & 0.12 & 0.18 & 0.1 & 0.07 \\
\hline Marzo & 0.14 & 0.05 & -0.12 & 0.1 \\
\hline Abril & 0.13 & -0.02 & 0.12 & 0.15 \\
\hline Mayo & 0.08 & 0.12 & -0.14 & 0.02 \\
\hline
\end{tabular}

\begin{tabular}{|c|c|}
\hline CARTERAS & CORRELACIONES \\
\hline BNV/ATK & -0.26502 \\
\hline BNV/BNK & 0.509321 \\
\hline BNV/GRNC & 0.491512 \\
\hline ATK/BNK & -0.01501 \\
\hline ATK/GRNC & -0.79241 \\
\hline BNK/GRNC & 0.406824 \\
\hline
\end{tabular}

Los datos que han sido procesados con la hoja de cálculo EXCEL arrojan que la cartera más riesgosa sería la combinación (BNV/ BNK) y la menos riesgosa la cartera (ATK/ GRNC). Afirmación que es fundamentada gráficamente.

\section{Correlaciones de las carteras}

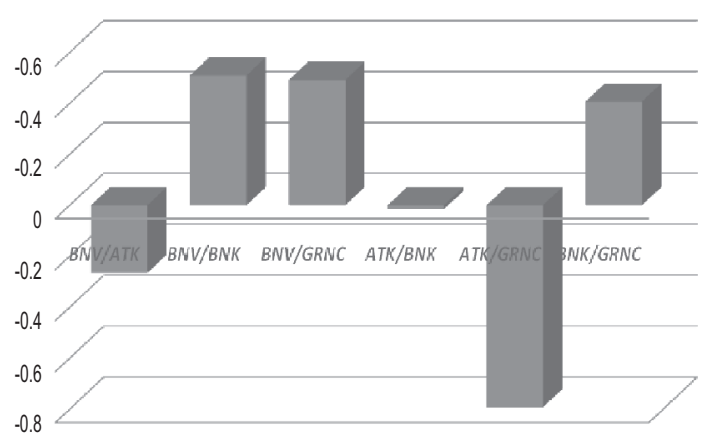

\section{CONCLUSIONES}

- La volatilidad de los mercados financieros exige la utilización de mayores herramientas económicas, estadísticas o matemáticas para tomar decisiones con altas probabilidades de acierto.

- La sensibilidad del precio del bono, el cual es medido por la desviación típica, depende de los tipos de interés del mercado, que es influenciado en su comportamiento por las políticas monetarias de los Bancos Centrales. 
- Si una acción es de elevada sensibilidad, proyectará una elevada desviación típica, y con ello los rendimientos esperados serán mayores. Asimismo, el nivel de riesgo, que es cuantificada por la desviación típica podría originar elevadas probabilidades de pérdidas, estos resultados dependeran del grado de volatilidad del activo financiero.

- Si un inversionista es adverso al riesgo, estructurará una cartera cuyo coeficiente de correlación se aproxime a -1 ; si le gusta el riesgo y quiere obtener máximas rentabilidades, optará por un cartera con un coeficiente de de correlación cercano $a+1$. Una perfecta cobertura implica op- tar por una cartera con un coeficiente de correlación igual a-1.

\section{REFERENCIAS BIBLIOGRÁFICAS}

1. Brealey, Richardy Myers, Stewart (2001). Principios de finanzas corporativas. Editorial McGraw-Hill, España.

2. Gitman, Lawrence J. (2000). Principios de administración financiera. Editorial Addinson Wesley, México.

3. Brigham, Eugene \& Houston Joel (1997). Fundamentals of financial management. Editorial The Dryden Press, USA.

4. Ross, S. y Westerfield (1997). Fundamentos de finanzas corporativas. Edit. Irwin, España. 